\title{
Bubble Column Reactor Fluid-dynamic Evaluation at Pilot-plant for Residue and Extra-heavy Crude Oil Upgrading Technology
}

\author{
Ricardo Sardella Palma ${ }^{1 *}$, Zacarías Luis ${ }^{2}$, Paiva Miguel $^{2}$ and Medina Henry ${ }^{1}$ \\ 1. Infrastructure and Upgrading Department, PDVSA-Intevep, Los Teques-Miranda 1201, Venezuela \\ 2. Refining Department, PDVSA-Intevep, Los Teques-Miranda 1201, Venezuela
}

\begin{abstract}
Bubble column reactors are multiphase contacting devices used in a wide variety of industrial application. Inrtevep S. A. is working on developing technologies to convert heavy and extra-heavy crude oil using this type of reactors. Volumetric gas hold up, flow pattern, average gas bubble size, average interfacial area, RTD (residence time distribution), dispersion coefficient, Péclet number are important design parameters for a proper scale up of them. Several cold model experiments have been proposed to determine the previously mentioned parameters at atmospheric conditions, using a plexiglas bubble column reactor at pilot plant scale unit (12 cm diameter). It was also evaluated our own design of internal trays (plates) in the reactor. Air-tap water and air-light oil systems have been used. A wide operating condition range was applied, superficial gas velocity between $0.5-10 \mathrm{~cm} / \mathrm{s}$, liquid flowrate between 15-65 1/h. Generally speaking, working without internal trays was found that gas hold up increase along the reactor and it was possible to identify heterogeneous bubble, transition and turbulent flow pattern areas for the air-light oil system. Average gas bubble size increase along the reactor at bubble regime from 2-5 $\mathrm{mm}$ but at turbulent regime, stay oscillating between 1-3 mm. Average interfacial area increases exponentially with superficial gas velocity at any reactor height, till $1,412 \mathrm{~m}^{2} / \mathrm{m}^{3}$ for the air-light oil system but, at bubble flow regime, the average interfacial area is lower than $100 \mathrm{~m}^{2} / \mathrm{m}^{3}$, which negatively impact the reactor performance. Internal trays in the reactor always increase gas hold up at any condition or system used. Residence time distributions curves, Péclet numbers and dispersion coefficients founded, show that this reactor with this kind of design internal trays still tends to be a complete mixing reactor under the operating conditions used.
\end{abstract}

Key words: Fluid dynamic, bubble column reactor, residue upgrading technology, trays.

\section{Introduction}

It is well known that bubble column reactor have a wide range of applications such as absorption, catalyst slurry reactor, bioreactions, coal liquefaction, etc. These reactors are preferred because the simplicity of its operation, easy construction and low operating costs. These main characteristics make bubble column reactors an excellent choice for extra-heavy oil upgrading technology as Aquaconversion ${ }^{\circledR}$.

Venezuela has extensive reserves of heavy and extra heavy crude oil in the eastern part of the country, including conventional production areas and the Orinoco Oil Belt. The Orinoco Oil Belt is divided in

\footnotetext{
*Corresponding author: Ricardo Sardella Palma, magister, research filed: fluid-dynamic. E-mail: sardellapr@pdvsa.com.
}

four big areas named as Carabobo, Ayacucho, Junín and Boyacá, and has 297,7 MMMBLS of proved reserves of extra heavy crude oil $77 \mathrm{MKm}^{2}$ approx. The Orinoco Oil Belt crude has very high densities (5-9 $9^{\circ}$ API (American petroleum institute) gravity) which reduce his market value and a very high viscosity $\left(10-40 \mathrm{Kcst}\right.$ at $\left.50{ }^{\circ} \mathrm{C}\right)$ which impact the transportation cost. This crude also has high sulfur (2-5w\%) and metal content (400-600 ppmw).

Production of these extra heavy crudes with gravities of $8-9^{\circ}$ is currently done by dilution with light crude $\left(24-30^{\circ}\right)$ in the well to be lifted to the surface, where it goes through a process scheme including degasification, dehydration and desalting to be commercialized as a dilute crude of $13.5-16^{\circ}$ API 
gravity and a viscosity less than $350 \mathrm{cst}$ at $50{ }^{\circ} \mathrm{C}$. The quantity of diluents could be around $38 \%-50 \% \mathrm{v}$ of the total production, depending on the light crude oil used. One of the main constraints in the current production of extra heavy crude in eastern Venezuela is the availability of light or medium crudes to be used as diluents.

This situation will be even worse in the future due PDVSA aim to incorporate more heavy and extra heavy crude oil in its crude portfolio to reach production capacity of 6 million BPD (Barrel per day) by 2019 . In order to overcome the constraints imposed by the potential shortage of light crudes required as diluent, PDVSA-Intevep has developed a new low-capital-cost technology for moderate conversion of residue and heavy or extra-heavy crude oil called Aquaconversion $^{\circledR}$.

Aquaconversion $^{\circledR}$ is a thermal catalytic steam cracking technology developed by PDVSA-Intevep to convert residue and heavy or extra heavy crude oil in the producing field into transportable upgraded crude, reducing the use of light/medium crude as diluents. The process is also utilized in petroleum refineries to increase conversion of vacuum residue without losing unconverted bottom-product stability [1].

In spite of the advantages, bubble column reactors used in a technology as Aquaconversion ${ }^{\circledR}$ are difficult to design and scale up, because of the flow regime complexity, and their unknown behavior under different sets of design parameters such a diameter, height, etc. Nonadjustable parameters like phase holdups, gas-liquid interfacial area, interfacial mass transfer coefficient, dispersion coefficient and heat transfer coefficient have a direct effect on the design of the bubble column reactor. Therefore, this study presents some experimental results to understand the fluid dynamic behavior of a bubble column at pilot plant scale, working under similar Aquaconversion ${ }^{\circledR}$ operating conditions of liquid and gas flow rates, but, using atmospheric conditions of pressure and temperature.

\section{Experimental Sections}

\subsection{Experimental Set Up}

A schematic diagram of the experimental apparatus is shown in Fig. 1. This unit was conformed by several sections. The liquid section had three tanks, one for recycling tests (gas hold up tests) another, for one through tests (dispersion coefficient test) and the last for disposal of liquid material. This section also contained the pumping system. The gas section capable of feeding air at required conditions, from the air pilot plant network into the unit has a pressure control valve, Brooks rotameter and an Endress Hauser mass flowrate meter used to accurately data measurement. In the reactor section was used a $12 \mathrm{~cm}$ diameter plexiglas column which is geometrically similar to the real Aquaconversion ${ }^{\circledR}$ reactor (see Fig. $2)$. The bubble column is a plexiglas tube with inner diameter of $12.3 \mathrm{~cm}$, the cylindrical height of $160 \mathrm{~cm}$, and a cone-shaped bottom also made of plexiglas without gas sparger. The special design cone-shape column top was made of metal and it was built as the real apparatus.

The column was equipped with a Rousemount 115 $T$ DPT (differential pressure transmitter) which is connected to ABB Screen Master 200 videografic register to gather the column pressure drop. This unit had a pneumatic liquid tracer system which is used to inject both tracers used in this study, Thiophene and Potassium Dichromate. Those injections were used to determine the dispersion coefficient in the column. All tests were conducted at room conditions of temperature and pressure.

Two different systems were used in this study, air-tap water and air-light oil. The light oil was chosen because of some physical and chemical properties like, density, surface tension and viscosity, which has to be similar to the feedstock (vacuum residue) at operating, conditions (180 psig of pressure and around $430{ }^{\circ} \mathrm{C}$ of temperature). These main liquid properties are shown in Table 1. 


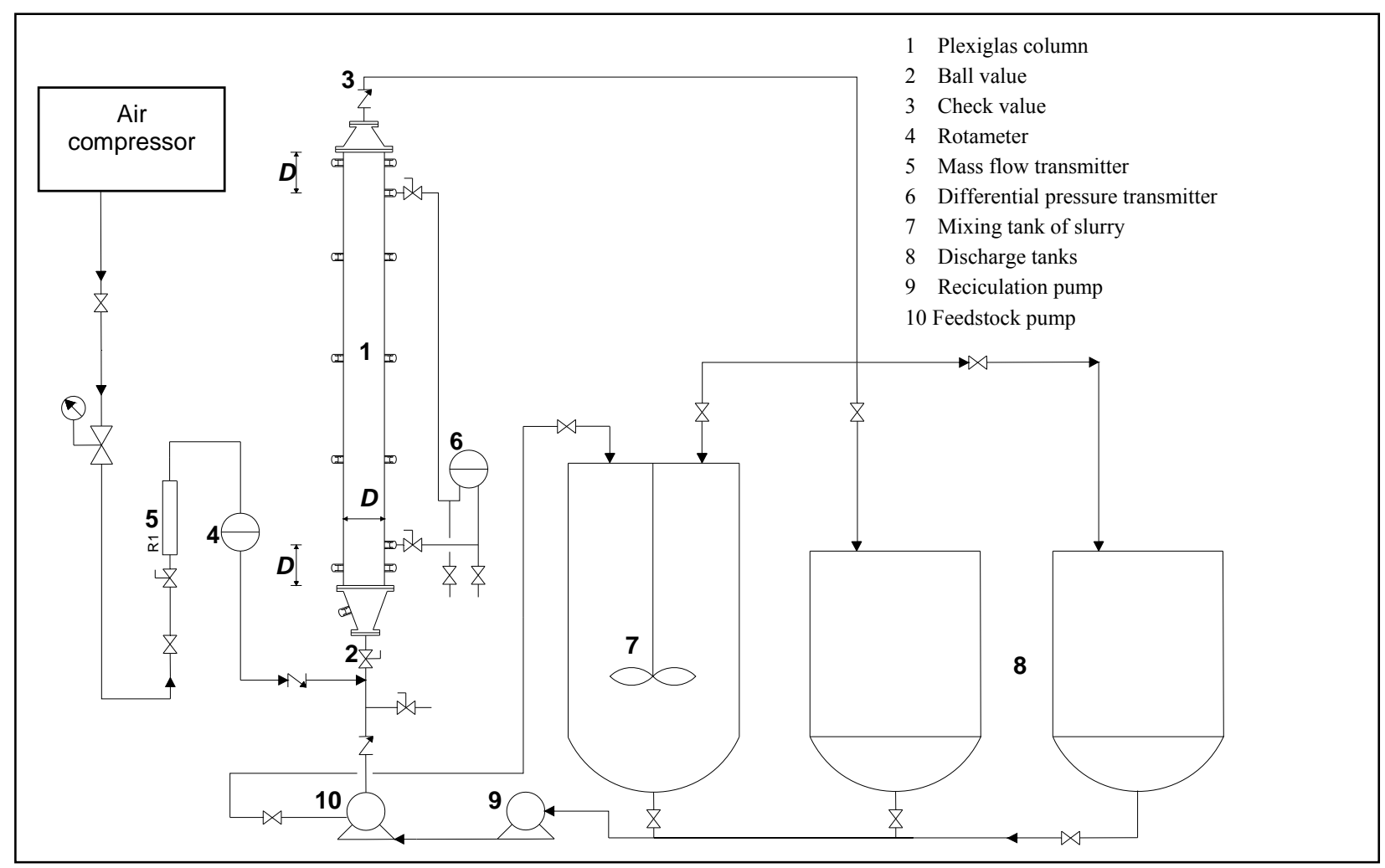

Fig. 1 Schematic diagram of the experimental apparatus.

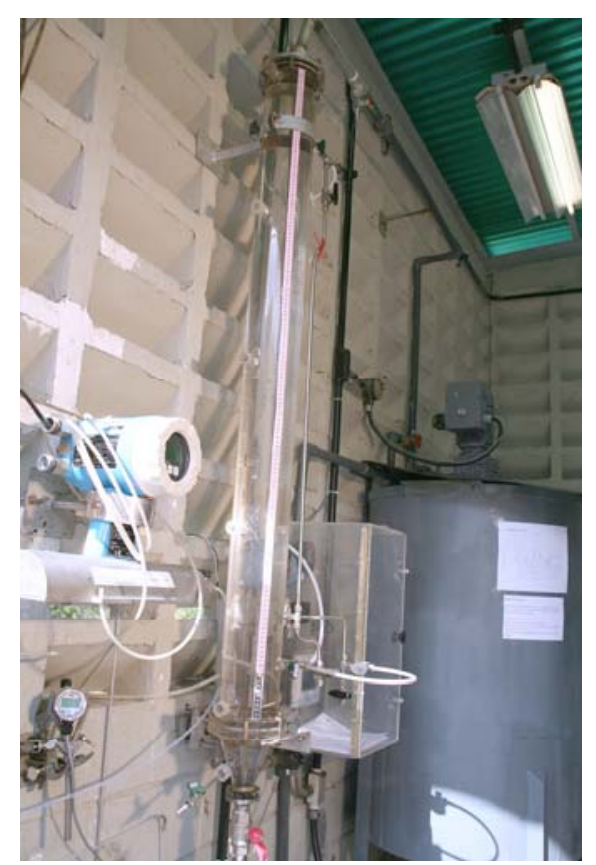

Fig. 2 Plexiglas reactor.

The upgrading process use steam as gas phase, but air was used because of availability and economic aspects. Table 2 shows a comparison of the properties of the two gases. Akita [2] used water and four gases of different densities, air, oxygen, carbon dioxide and helium in a column with $15 \times 15 \mathrm{~cm}$ square cross section, showed that the effect of the gas density on the gas hold up could be neglected. Nevertheless, in very high pressure systems, in which the gas density can be affected in a higher proportion, the gas hold up, could increase significantly with the increase of the gas density [3].

A wide operating condition range was used, in order to reproduce the actual conditions of the pilot plan unit. Superficial gas phase velocities between $0.5-10 \mathrm{~cm} / \mathrm{s}$ and liquid flowrate between $15-65 \mathrm{l} / \mathrm{h}$ (liquid phase velocity between $0.035-0.15 \mathrm{~cm} / \mathrm{s}$ ). Three different flowrates were used, 15, 35 and $651 / \mathrm{h}$ and for each one, a set of superficial gas velocity were tested from $1 \mathrm{~cm} / \mathrm{s}$ to $10 \mathrm{~cm} / \mathrm{s}$ with $1 \mathrm{~cm} / \mathrm{s}$ steps. In some cases, it was necessary to operate at $0.5 \mathrm{~cm} / \mathrm{s}$ of superficial gas velocity.

It was tested internal trays inside the column in order to define their impact on the gas hold up and liquid axial dispersion coefficient. The internal trays 
Table 1 Liquid phase properties.

\begin{tabular}{lllll}
\hline Fluid & Method & $\begin{array}{l}\text { Viscosity } \\
(\mathbf{c s t})\end{array}$ & Density $\left(\mathbf{K g} / \mathbf{m}^{3}\right)$ & Surface tension (dyn/cm) \\
\hline Vacuum residue & PROII & 1.7 & 843 & 16.63 \\
Light oil & Whihelmy plate & $\begin{array}{l}2-3.5 \text { at } 40{ }^{\circ} \mathrm{C} \text { ASTM } \\
(\mathrm{D}-445)\end{array}$ & $\begin{array}{l}815 \text { at } 25^{\circ} \mathrm{C} \text { ASTM } \\
(\mathrm{D}-1298)\end{array}$ & 27.36 at $25^{\circ} \mathrm{C}(\mathrm{Lab} . \mathrm{E} \& \mathrm{P})$ \\
Water & Perry, 1992 & 1.00 & 996 & 72.8 \\
\hline
\end{tabular}

Table 2 Gas phase properties.

\begin{tabular}{lllll}
\hline Fluid & $\begin{array}{l}\text { Viscosity } \\
\text { (cst) }\end{array}$ & $\begin{array}{l}\text { Mol. } \\
\text { weight }\end{array}$ & Density $\mathbf{( K g / \mathbf { m } ^ { 3 } )}$ & Surface tension $(\mathbf{d y n} / \mathbf{c m})$ \\
\hline Steam & 0.025 & 18 & 3.69 & 4.93 \\
Air & 0.019 & 29 & 1.18 & 1.18 \\
\hline
\end{tabular}

consist on 10 perforated trays with $3 \mathrm{~mm}$ diameter holes. (Fig. 3). The number of holes change, at the bottom is around 232 holes and increases along the column till reach 264 holes at the top, in order to keep gas velocity constant in the column trough the holes because the gas formation inside the column when this internal tray is used inside the real reactor, following typical shell internal trays design used in visbreaking commercial reactor.

\subsection{Methods}

This part consists of the description of the procedure used to estimate gas hold-up, flow pattern, dispersion coefficient and average gas bubble size and average interfacial area.

\subsubsection{Gas Hold Up}

The unit was operating in recycling mode. Two different methodologies were used, the first one consist on determining the pressure drop in the column and the second methodology was based on phase trapping and measure both phases inside the column. The pressure drop is determined by using a DPT in the column. The differential pressure profile is deduced from a one-dimensional biphasic model, where friction losses and fluid acceleration are neglected. Then, the axial pressure profile in the $\mathrm{c}$ olumn can be described by the Eq. (1). Finally, with Eqs. (1) and (2), it is possible to define a total gas and liquid hold ups in the section where the pressure drop is determined. This procedure was done in order to

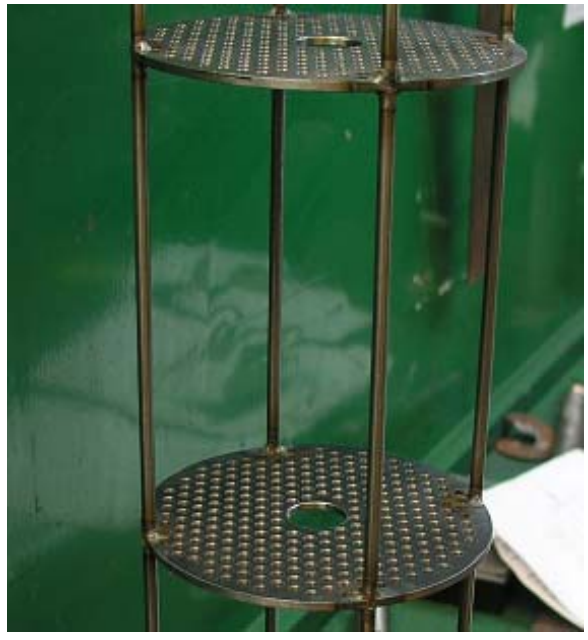

Fig. 3 Internal trays tested.

determine the total and partial (top and bottom sections) hold up in the column. Both sections represent almost half column.

$$
-\frac{d P}{d z}=\left(\varepsilon_{L} \rho_{L}+\varepsilon_{G} \rho_{G}\right) * g
$$

where, $d P$ is the pressure differential, $d z$ is the height differential, $\varepsilon_{L}$ and $\varepsilon_{G}$ are the liquid and gas holds up, respectively and $g$ is the gravitational constant.

$$
\varepsilon_{L}+\varepsilon_{G}=1
$$

The phases trapping methodology consists on stopping both liquid and gas flow rate sent into the column and closing the inlet and outlet reactor valves to measure the height of both phases in the cylindrical section of the column. Then, it is possible to determine total gas hold-up as a relation between the gas volume and the total column volume, as it is shown in Eq. (3): 


$$
\varepsilon_{G}=\frac{V_{c-t o p}+V_{h}}{V_{T}}
$$

where, $V_{c \text {-top }}, V_{h,}, V_{T}$ are the volume of the cone at the top of the column, the obtained volume at specific height and the total column volume, respectively.

\subsubsection{Flow Regime}

A pneumatic system was used to inject into the reactor a pulse of a liquid tracer. Thiophene and Potassium Dichromate were used as tracer for air-light oil and air-tap water systems, respectively. When the pulse is injected, the systems stop working in recycle mode and the discharge is sent to the disposal liquid tank. Reactor outlet is sampled along the time in order to quantify tracer concentration; sulfur concentration (Tiophene) by WD-FRX using ASTMD-2620-10 norm and Potassium concentration (Potassium Dichromate) by ICP (induce coupled plasma). Injections were used to determine the RTD (residence time distribution curve) for all the cases. Levenspiel [4] defined a correlation between Péclet number and the RTD curve, as follows:

$$
\frac{\sigma^{2}}{\bar{t}^{2}}=\frac{2}{P e}+\frac{8}{P e^{2}}
$$

where, $P e$ is the Péclet number, $\sigma$ is the variance and $t$ is the average time.

Average time can be determined using the next Eq. (5) [4]:

$$
\bar{t}=\frac{\sum_{i=1}^{n} t_{i} C_{i} \Delta t_{i}}{\sum_{i=1}^{n} C_{i} \Delta t_{i}}
$$

where, $C_{i}$ is concentration of the component, $\Delta t$ is the time different between samples, and $t_{i}$ is time.

In the same way, variance can be determined by the following Eq. (6) [5]:

$$
\sigma^{2}=\frac{\sum_{i=1}^{n}\left(t_{i}-\bar{t}\right)^{2} C_{i} \Delta t_{i}}{\sum_{i=1}^{n} C_{i} \Delta t_{i}}
$$

Finally, dispersion coefficient can be obtained by the correlation commonly used in reactor design between the axial Péclet number based on the liquid superficial velocity and the dispersion coefficient [6]:

$$
P e=\frac{Q L}{A_{R} D}=\frac{u L}{D}
$$

where, $u$ is the liquid lineal velocity, $L$ is the column length tangent-tangent, $D$ is the dispersion coefficient.

The previous procedure was applied at different operating conditions: liquid flow rate of $35 \mathrm{l} / \mathrm{h}$ and three different superficial gas phase velocities $0.5,3$ and $6 \mathrm{~cm} / \mathrm{s}$.

2.2.3 Average Gas Bubble Size and Average Interfacial Area

It was used the DSD (dynamic gas disengagement) methodology to determine the average gas bubble size distribution [7]. This method is based on determining the average gas bubble size distribution when the gas bubble is disengaging of the gas-liquid bed inside the column. This measurement can be liked with the rising bubble velocity of different groups of bubble sizes. These velocities could be used to determine the average bubble size of each group of bubbles and then it is possible to estimate the Sauter diameter and the average interfacial area.

First, it is necessary to plot the bed height level as function of time when the gas inlet is closed by the following Eq. (8):

$$
\frac{H_{(t)}}{H_{0}}=\frac{\varepsilon_{G}\left(V_{c-\text { inf }}+A . H_{s}\right)}{H_{0}\left(100-\varepsilon_{G}\right) A}+\frac{H_{s}}{H_{0}}
$$

where, $H_{o}$ is the bed level before closing the gas inlet, $H_{(t)}$ is the bed level any time, $H_{s}$ is the bed level at the end, $A$ is the columns transversal area, and $V_{c-\text { inf }}$ is the inlet cone volume.

According to the shape of the curve obtained, the gas bubble sizes have to be defined as bi, trio, poly modal different sizes of groups of bubbles: small, medium and large group of bubble. For instance, each group of bubbles should be defined as a section of the curve obtained and represented by a straight line. The slope $\left(S_{i}\right)$ and the y-axis cut point $\left(b_{i}\right)$ should be 
determined (Fig. 4).

Then, gas bubble hold up for each individual group of bubbles could be determined for as follows:

$$
\varepsilon_{G i}=\frac{H_{s}}{H_{0}}\left[\frac{1}{b_{i+1}}-\frac{1}{b_{i}}\right]
$$

where, $b_{i}$ is the y-axis cut point of each group of bubble.

And the following equations could be used to estimate the gas bubble rising velocities:

$$
u_{b i}=\frac{H_{o}\left[b_{i} S_{i+1}-b_{i+1} S_{i}\right]}{b_{i}-b_{i+1}}
$$

where, $S_{i}$ is the curve slope of each group of bubble.

For small bubble, which is normally used the terminal rising velocity $\left(u_{b \infty}\right)$ based on a single bubble in an infinite medium determined by Marruci equation [7]:

$$
u_{b \infty}=\frac{u_{b S}\left[1-\varepsilon_{G S}^{5 / 3}\right]}{\left(1-\varepsilon_{G S}\right)^{2}}
$$

where, $u_{B s}$ is the small bubble velocity.

Then, average gas bubble size diameter for each group of bubbles could be determined following Stokes's law, or Peebles and Garber, Clift or Abou-el-Hassan equations as it is shown in Table 3.

Finally, to estimate the Sauter diameter $\left(d_{S}\right)$ of the bubble and the average interfacial area $\left(a_{S}\right)$ can be obtained by the next two Eqs. (12) and (13) [7]:

$$
\begin{gathered}
d_{S}=\frac{\sum_{i=1}^{n} n_{i} d_{b}^{3}}{\sum_{i=1}^{n} n_{i} d_{b}^{2}}=\frac{\varepsilon_{G}}{\sum_{i=1}^{N} \varepsilon_{G i} / d_{b i}} \\
a_{S}=\frac{6 \varepsilon_{G}}{d_{S}}=6 \sum_{i=1}^{N} \frac{\varepsilon_{G i}}{d_{b i}}
\end{gathered}
$$

where, $\rho_{L}$ and $\rho_{G}$ are liquid and gas density, $\mu_{L}$ is the liquid viscosity and the $\sigma \mathrm{L}$ is the liquid surface tension.

\section{Results and Discussion}

To ensure ssystem stability and quality of the results,

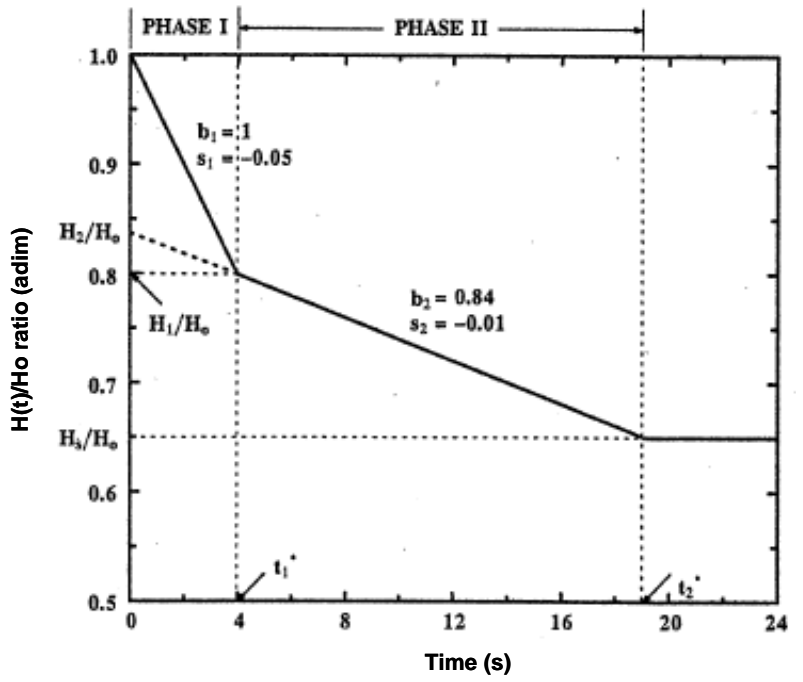

Fig. 4 Gas disengagement profile on time.

Table 3 Bubble size diameter equations.

\begin{tabular}{ll}
\hline Reference & Correlation \\
\hline Stokes's law & $d_{b S}=\left[\frac{18 \mu_{L} u_{B}}{g\left(\rho_{L}-\rho_{G}\right)}\right]^{0.5}$ \\
Peebles and Garber & $d_{b S}=4.76\left[\frac{\mu_{L}}{\rho_{L}}\right]^{0.41} \frac{u_{B}^{0.78}}{g^{0.59}}$ \\
Clift & $u_{b S}=\left[\frac{2.14 \sigma_{L}}{\rho_{L} d_{B}}+0.505 g d_{B}\right]^{0.5}$ \\
& $V=0.75[\log (F)]^{0.5}$ \\
Abou-el-Hassan & $V=\frac{u_{B}\left(d_{B} \rho_{L}\right)^{2 / 3}}{\left(\mu_{L} \sigma_{L}\right)^{1 / 3}}$ \\
& $F=\frac{g d_{B}^{8 / 3}\left(\rho_{L}-\rho_{G}\right) \rho_{L}^{2 / 3}}{\mu_{L}^{4 / 3} \sigma_{L}^{1 / 3}}$ \\
\hline
\end{tabular}

column pressure drop was monitored and registered for all sets of experiments done here. For all the experiments conducted, the transient period of the system was less than $5 \mathrm{~min}$. It was reached operational stability after that time.

\subsection{Gas Hold Up Profile and Flow Patterns}

Fig. 5 is a plot of the experimental gas hold up and gas velocity in the bubble column reactor for the air-light oil system at $35 \mathrm{l} / \mathrm{h}$ of liquid flow rate at different superficial gas velocities (line with solid triangles). Gas hold up increases when superficial gas velocity increases, as expected, following a potential shape curve ( $\varepsilon=a U g^{N}$ type) described by Shah [8]. 


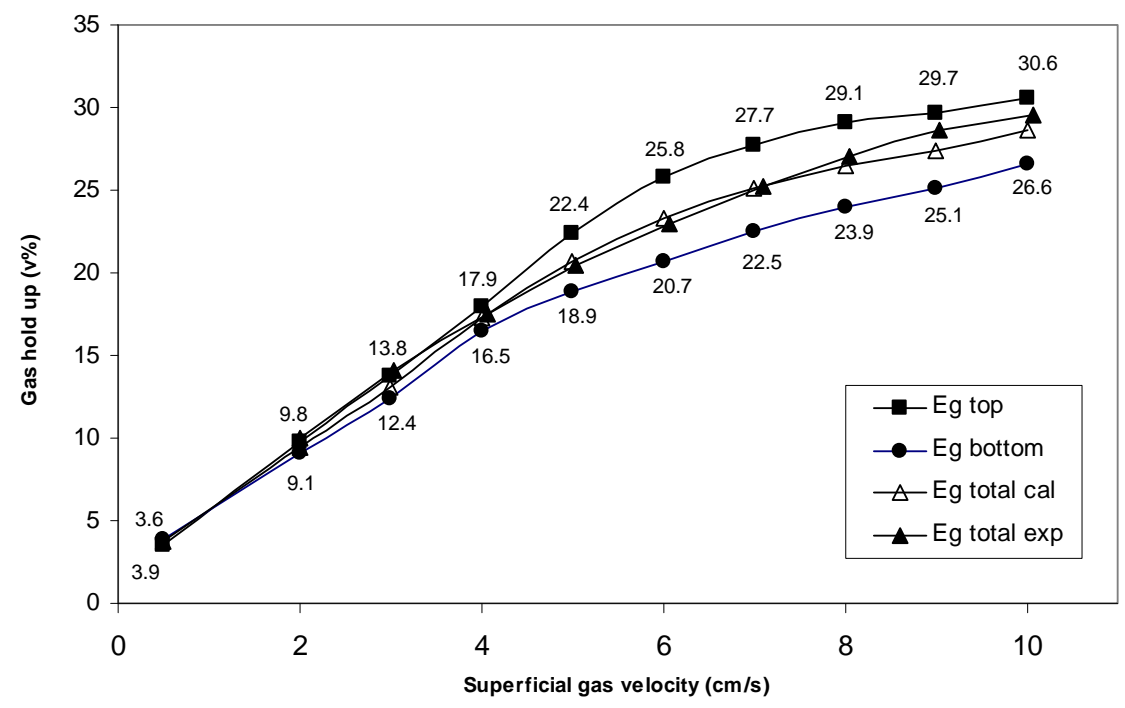

Fig. 5 Gas hold up for air-light oil system at $35 \mathrm{l} / \mathrm{h}$ of liquid flow rate.

The equation found for the air-light oil system was $\varepsilon=$ $0.0821 \mathrm{Ug}^{0.5493}$. According to Shah, if the exponential number is lower than 0.7 , in this case, 0.5493 , then the flow pattern tend to be church turbulent.

Empty triangles line represents the calculated total gas hold up obtained using the pressure drop methodology based on both, top and bottom gas hold up by partial pressure drops, which was exactly the same value obtained when the total gas hold up was determined by the pressure drop methodology for the total column. All the results showed in this work are based on pressure drop methodology used. But, the difference between the phases trapping and the pressure drop methodologies was only of $2.9 \%$ on average [9].

Top and bottom sections gas hold up are shown at the Fig. 5 on square and cycle lines, respectively. It can be seen that gas hold up at the top of the column is almost always higher than the gas hold up at the bottom. The differential between both curves increase when the superficial gas velocity is higher than $2 \mathrm{~cm} / \mathrm{s}$ and it is even higher after $4 \mathrm{~cm} / \mathrm{s}$.

Only at superficial gas velocity of $0.5 \mathrm{~cm} / \mathrm{s}$, the gas hold up at the top and bottom are quite de same. This result confirm what was observed by Sardella [9] where at $0.5 \mathrm{~cm} / \mathrm{s}$ of superficial gas velocity using the same condition and system it was defined the flow pattern as heterogeneous bubble flow, where different size bubbles rise along the reactor almost vertically without changing significantly bubbles volume or with a bubble size increasing to the pressure drop on the column.

After $2 \mathrm{~cm} / \mathrm{s}$ of superficial gas velocity, the gas hold up at the top of the column is clearly higher than the one at the bottom. As is mentioned by Sardella [9], after $2 \mathrm{~cm} / \mathrm{s}$ of superficial gas velocity, the visual flow pattern can be clearly defined as churn turbulent because it can be seen different size bubbles which change the volume along the column, with random movements, back flow and even axial swirl, with the big "jet" type bubble at bottom of the column which disappear along the column breaking down in small one.

The results obtained in this paper support the previous explanation because, big bubbles rise faster than small one, and faster the bubble lower the resident time in the column decreasing the gas hold up. Billet [10] found similar results.

After $4 \mathrm{~cm} / \mathrm{s}$ of superficial gas velocity the different between gas hold up at the top and bottom increase. This can be explained based on Sardella [9] where it was identify foam formation at the top of the column and due to the increasing of gas velocity, the big type 
"jet" bubble at the bottom of the column increase.

These two phenomena could explain the results obtained in this paper. Pino and Guitian found similar results with foam formation $[11,12]$.

\subsection{Average Gas Bubble Size and Average Interfacial Area}

Fig. 6 shows the bed height level as function of time when the gas inlet valve to the column is closed. This result was obtained working at $6 \mathrm{~cm} / \mathrm{s}$ of superficial gas velocity and with an initial liquid level of $155 \mathrm{~cm}$ in the column (total column). Similar behavior was obtained for the rest of the cases evaluated. As it was mentioned at the methodology section, it was necessary to split the curve in different lineal segments and categorize it as big, medium (if apply) and small bubbles.

During the test, it was observed that bigger the bubbles faster it disengages from the liquid. Finally the bed height level remains constant and equal to the beginning.

Fig. 7 shows the average gas bubble size using air-light oil system at three different heights of the column with 4 different superficial gas velocities: 0.5 , $1.5,3$ and $6 \mathrm{~cm} / \mathrm{s}$.

First, it is important to point out that the average gas bubble size decreases when the superficial gas velocity increases, at any column level. Similar results were obtained by Daly [7], using a $0.23 \mathrm{~cm}$ diameter column working with similar gas-liquid systems. These results confirm also that the bubble breaking phenomena is governing along the column when the gas flow rate is increasing, which is also more obvious because the "jet" type big bubble formation at bottom of the column. This is a typical phenomenon in a church turbulent flow regime.

Morsi [13] obtained an average bubble diameter of 1.5-3.5 mm, Shah [8] obtained 1-2 $\mathrm{mm}$ and Daly [7] obtained bubble diameter of 2-5 $\mathrm{mm}$. Those values are similar to the ones obtained in this work.

As it was mentioned before, at $0.5 \mathrm{~cm} / \mathrm{s}$ of superficial gas velocity, the average bubble size tend to increase along the column due to the pressure drop confirming the heterogeneous bubble flow pattern due to the coalescence phenomena. In spite of the average bubble diameter also increase along the reactor at $1.5 \mathrm{~cm} / \mathrm{s}$, typical of heterogeneous bubble flow pattern, according with Sardella [9] this condition was defined as transition zone which make very difficult to understand. After 3 $\mathrm{cm} / \mathrm{s}$, at the upper size of the column, it can be observed a breaking-coalescence bubble phenomena and the bubble diameter try to keep almost constants,

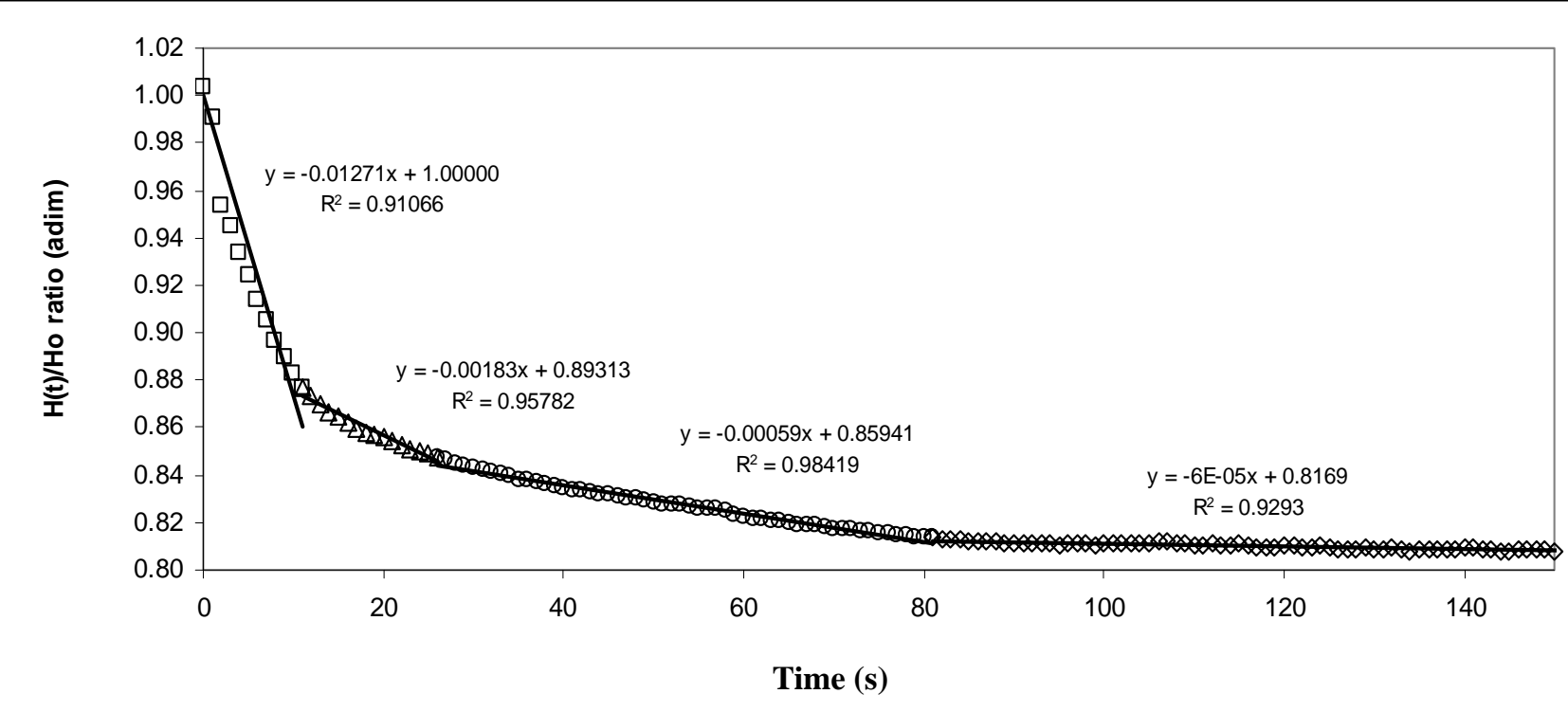

Fig. 6 Typical gas disengagement profile on time air-light oil system at $6 \mathrm{~cm} / \mathrm{s}$ and $155 \mathrm{~cm}$ of height. 


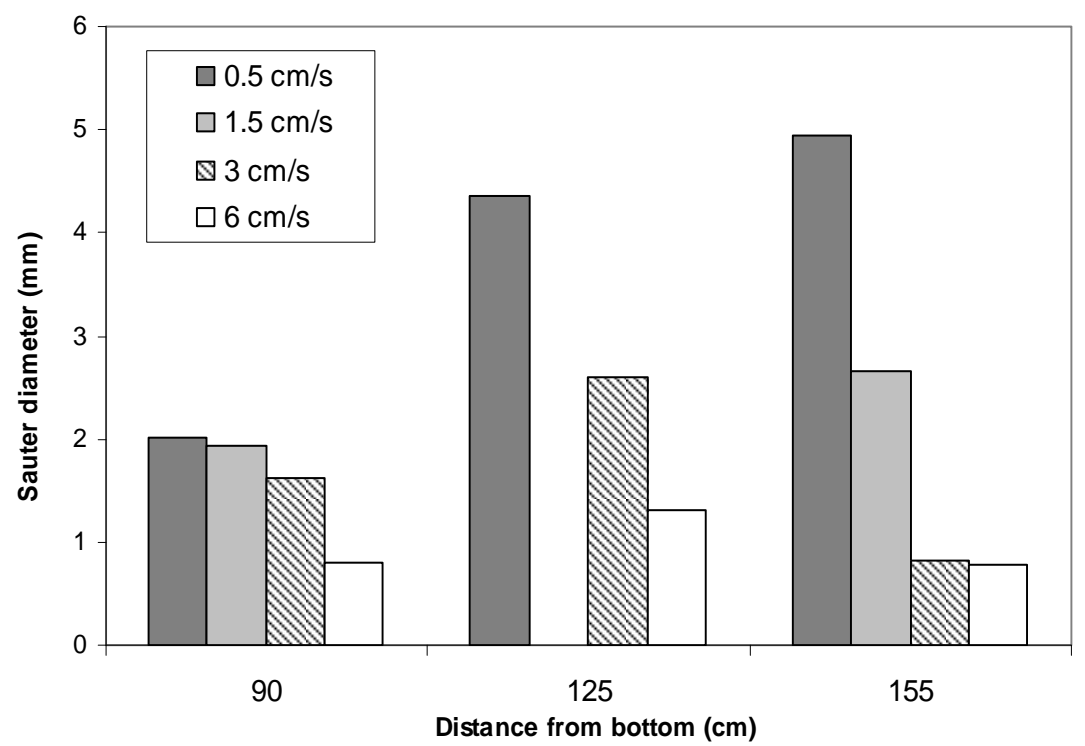

Fig. 7 Sauter diameter for air-light oil system at different superficial gas velocity.

increasing and decreasing just a little, which is a typical behavior of a churn turbulent flow pattern. At $155 \mathrm{~cm}$ of liquid height level, it can be seen also that the average bubblediameter tend to reach a constant value, after $3 \mathrm{~cm} / \mathrm{s}$, which also can explain the tendency to reach minimum constant value in the gas hold up at the top of the column shown in the Fig. 5, because it reached a dynamic equilibrium between breaking and coalescence phenomena. Those results also confirm the flow patterns defined by Sardella [9] and the gas hold up profile already shown in this paper.

As it is mentioned on Soong [14] Akita y Yoshida proposed an equation to estimate average bubble diameter $d_{32}$, to be used on column till $30 \mathrm{~cm}$ of diameter, and superficial gas velocity lower than 7 $\mathrm{cm} / \mathrm{s}$, based also on physical-chemical properties and column diameter as it is following shown:

$$
d_{32}=26 D_{C}(B o)^{-0.5}(G a)^{-0.12}(F r)^{-0.12}
$$

The Fig. 8 shows that, in spite of the value are not exactly the same, the tendency of the average bubble diameter $\left(d_{32}\right)$ as a function of the superficial gas velocity are very similar, and the Akita and Yoshida equation could be used to estimate the average bubble diameter in this column working with this systems.
The comparison was made using the average bubble diameter experimentally obtained at $155 \mathrm{~cm}$ of liquid height in the column, because it almost represents the whole column scenario. Fig. 8 also shows that the average bubble diameter tends to reach a minimum constant value at higher superficial gas velocities as it was commented previously.

The gas-liquid interfacial area is a very important variable which is impacted by the column geometry, the operating conditions and liquid properties. The gas-liquid interfacial area is very important in this kind of reactor because it could impact the mass transfer. Fig. 9 shows the gas-liquid interfacial area at different superficial gas velocities working a liquid flow rate of $35 \mathrm{l} / \mathrm{h}$ at several liquid levels in the column.

Due to the fact the gas-liquid interfacial area depend on the gas hold up and the average bubble diameter as it is shown in the Eqs. (12) and (13), higher the superficial gas velocity, higher the gas hold up and lower the average bubble diameter, impacting almost exponentially the gas-liquid interfacial area as it is shown in the Fig. 9 at any liquid level. Previous works have found similar gas-liquid interfacial area (900-1200 $\mathrm{m}^{2} / \mathrm{m}^{3}$ ) working with inorganic liquid and with superficial gas velocities between $3-14 \mathrm{~cm} / \mathrm{s}$ [15]. 


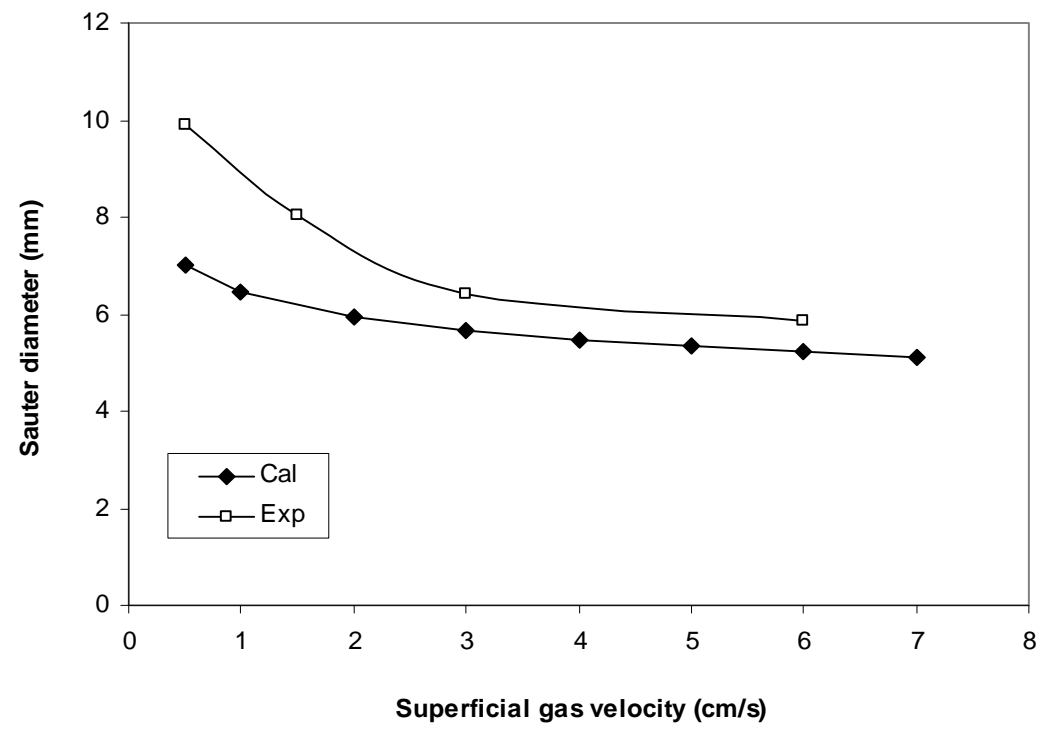

Fig. 8 Bubble diameter comparison with Akita and Yoshida correlation.

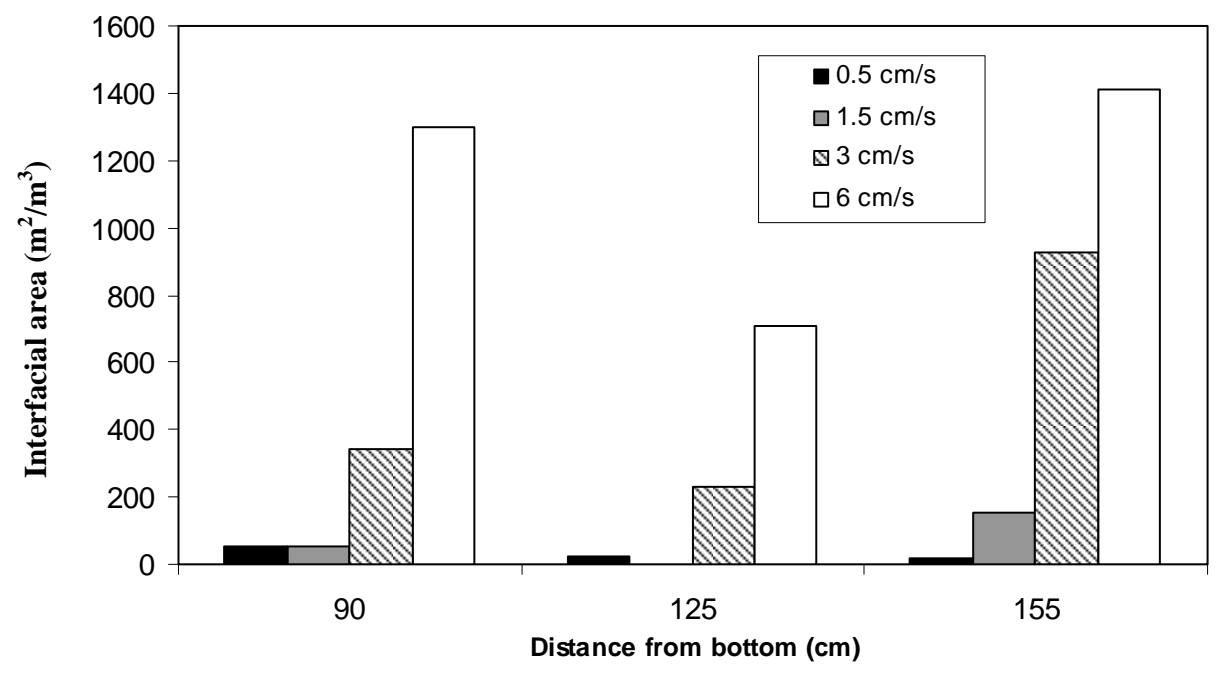

Fig. 9 Interfacial area for air-light oil system.

Working at heterogeneous bubble flow pattern at $1.5 \mathrm{~cm} / \mathrm{s}$, the gas-liquid interfacial area obtained is very low (lower than $150 \mathrm{~m}^{2} / \mathrm{m}^{3}$ ), which could negatively impact the mass-transfer coefficient (Kla), with a potential decreasing of the vacuum residue conversion at the real reactor, dropping the reactor performance. Working at church turbulent flow pattern, at $3 \mathrm{~cm} / \mathrm{s}$ or $6 \mathrm{~cm} / \mathrm{s}$, the situation change dramatically and the gas-liquid interfacial area increase till the expected value for this kind of moderate hidroconversion reactor. As reference, according to Trambouze [16], the gas-liquid interfacial area for moderate hidrotreatment process should be around $200-1,000 \mathrm{~m}^{2} / \mathrm{m}^{3}$.

Fig. 10 shows the comparison between the air-tap water and the air-light oil systems, working at 155 of liquid level and at three different superficial gas velocities. As it can be seen, the average bubble diameter of the air-tap water system is always higher (at least 4 times) than the air-light oil system.

The tendency was very similar to the one found with light oil. The average bubble diameter is higher 


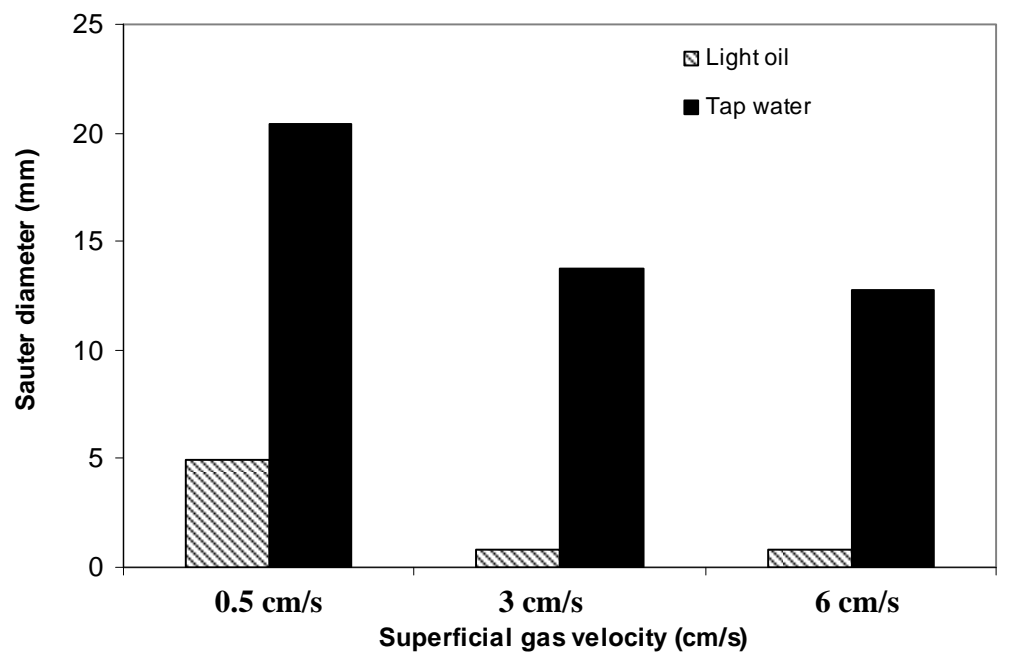

Fig. 10 Air-tap water and air-light oil systems Sauter diameter comparison.

at bubble flow pattern than the one found at turbulent flow pattern. This result can be explained because as it is shown in Table 1, light oil has lower surface tension than the water. According to Lemoine [15] and Daly [7], when liquid surface tension decreases, the system become less coalescing and the gas hold up increase. This was observed by Sardella [9]. The lower the liquid surface tension, the lower the bubble size, increasing the interfacial area and making the system easier to transport. Smaller bubble produce larger gas holds up due to their low rise velocities. Kelkar and Daly found similar results $[7,17]$.

Daly [7] found average bubble diameter between 2-5 $\mathrm{mm}$ for tap water systems, but Lemoine [15] found values around 5-9 $\mathrm{mm}$ for the same system in a column of $7.8 \mathrm{~cm}$ diameter on church turbulent flow regime, which is more similar to the values obtained in this work.

\subsection{Reactor Trays Evaluation}

\subsubsection{Reactor Trays Evaluation}

Fig. 11 shows the gas hold up with tap water-air and light oil-air systems at a liquid flow rate of $35 \mathrm{l} / \mathrm{h}$ at different superficial gas velocities with and without trays inside the column. As it can seen, trays inside the column increase clearly the gas hold up in both systems tested. Trays inside the column make the system less coalescing, breaking more the bubbles, creating small bubbles. As it was mentioned before, smaller bubble produce larger gas holds up due to their low rise velocities. Kelkar and Daly found similar results [7, 17].

Alvare [18] found that trays inside the column also reduce the liquid recirculation which then, reduces its hold up.

\subsubsection{Flow Regime}

Fig. 12 shows the obtained Residence Time Distribution curves for the air-light oil system working at $35 \mathrm{l} / \mathrm{h}$ of liquid flow rate and three different superficial gas velocities $(0.5,3$ and $6 \mathrm{~cm} / \mathrm{s})$ with internal trays inside the column. In order to compare all the systems it was necessary to normalize the RTD curves, constructing which is normally called E curves. It can be seem that all curves follow the same shape. They have a peak at the beginning and then fall progressively. These curves are typical for well mixed flow regime systems. The higher the superficial gas velocities, the higher the tracer concentrations, because the liquid hold up is lower. The difference on time between all the peaks is very small, but, it is possible to identify that at higher gas velocity, the peak is found faster, as expected.

Table 4 shows dispersion coefficients and Péclet numbers found for air-light oil system at $35 \mathrm{l} / \mathrm{h}$ of 


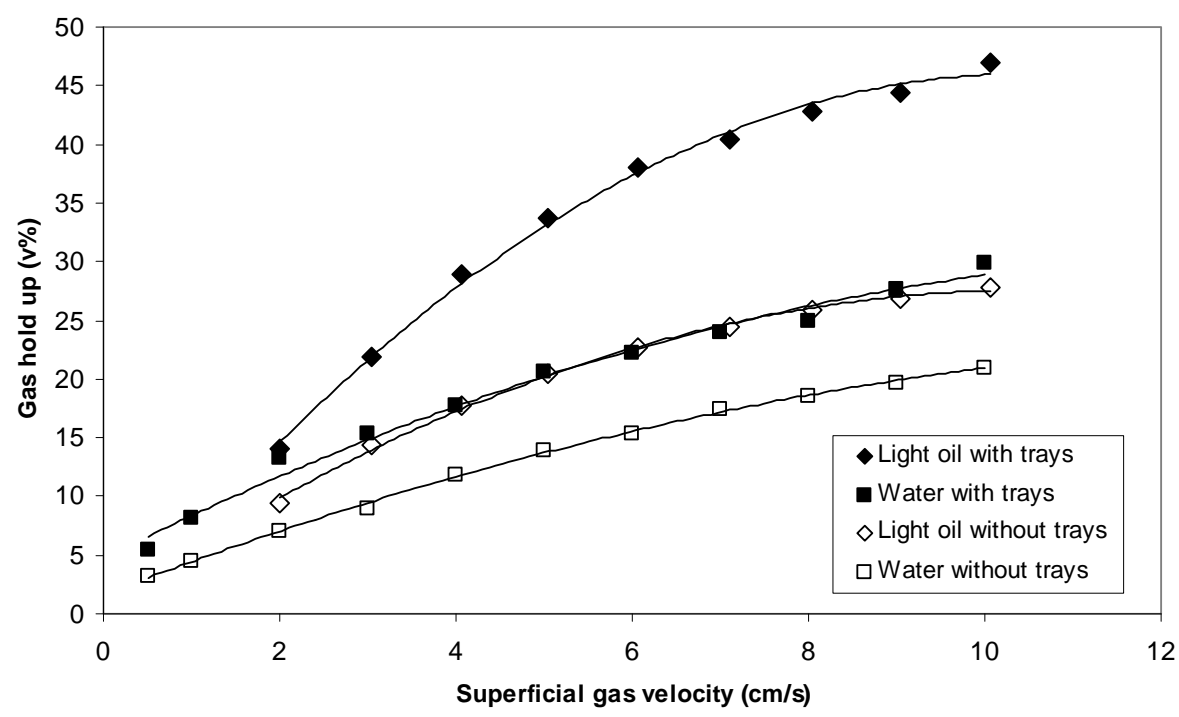

Fig. 11 Gas hold up with and without trays.

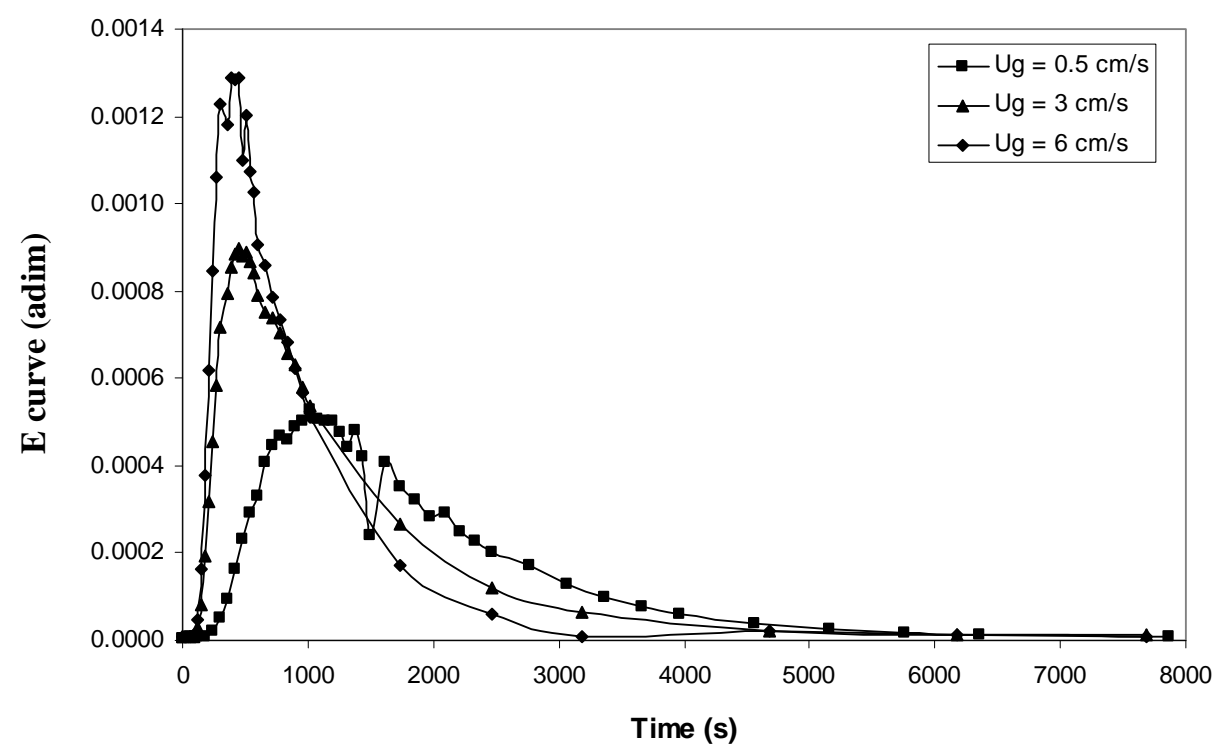

Fig. 12 E curves for air-light oil systems at $35 \mathrm{l} / \mathrm{h}$ of liquid flow rate with trays.

Table 4 Flow regime parameter for air-light oil system at $35 \mathrm{l} / \mathrm{h}$ of liquid flow rate.

\begin{tabular}{llll}
\hline Superficial gas velocity $(\mathbf{c m} / \mathbf{s})$ & $\begin{array}{l}\text { Mean time } \\
\text { (s) }\end{array}$ & Dispersion coefficient $\left(\mathbf{c m}^{2} / \mathbf{s}\right)$ & Péclet number (adim) \\
\hline 0.5 & 1,970 & 12.6 & 6.42 \\
3 & 1,476 & 111.6 & 4.30 \\
6 & 1,130 & 304.8 & 3.15 \\
\hline
\end{tabular}

liquid flow rate and three different superficial gas velocities. It can be seen that for all the cases, similar and small Péclet number values and similar and high dispersion coefficient values. Based on the Péclet numbers and the RTD curves, it is possible to define that this reactor at $35 \mathrm{l} / \mathrm{h}$ of liquid flow rate and any gas velocities tends to be a well mixed flow reactor. Carberry [6]. Similar results were found for the air-tap water system.

Fig. 13 shows a comparison with and without 
internal trays inside the column working at $35 \mathrm{l} / \mathrm{h}$ of liquid flow rate and $3 \mathrm{~cm} / \mathrm{s}$ of superficial gas velocity, using Sardella [9] results. Both E curves have the same behavior, but due to the higher gas hold up on obtained with the internal trays, tracer concentration increases inside the column and the peak value in this system is higher than the value found for the column with internal. When it is used internal trays inside the column the peak of the tracer concentration appears later on time, almost the double of the time due to the increasing on the number of the mixing stages creating by the trays.

It was found a Péclet number of 4.30 is the same to the one obtained without internal trays Sardella [9], reason why this system also can be defined as a complete mixing reactor.

Another way to see the results is trough the F curves (Fig. 13), which represent the quantity of tracer removed as function of time. For the system with internal trays, the quantity of tracer removed out the reactor at the average residence time $(2,119 \mathrm{~s})$ is between $84 \%$, around $10 \%$ more than the quantity of material obtained without trays, but in both cases the tracer remain inside the column until $8000 \mathrm{~s}(2 \mathrm{~h}$ and $13 \mathrm{~min}$ ), almost four times the average residence time defined for the liquid flow rate. This result indicate that the tray inside the column could improve products selectivity, because, less material remain in the reactor after at the desired residence time. At operating condition with the vacuum residue then, this situation could decrease coke formation.

The results obtained with the internal trays shows that dead volumes are obtained by mean of the internal trays, displacing the peak of the curve on time, without changing the flow regime, as it is mentioned on Fogler [19].

Alvare [18] explain that the total free area of the holes should be lower than $10 \%$ of the total area of the tray in order to obtain an important change in the flow regime. Trays tested in this work have $14-16 \%$ of free area, which is higher the value mentioned at Alvaré [18], and it could be the reasons of the results obtained in this work. Dead zones are very common on fixed bed reactor, nevertheless, trays can also create dead zone just over it. Higher the superficial gas velocity, higher the backflow mixing between each pair of trays, reducing potential dead zone, making the RTD more similar to the one obtained without internal trays. Dead zone are volume with negligible velocities which could promote coke formation.

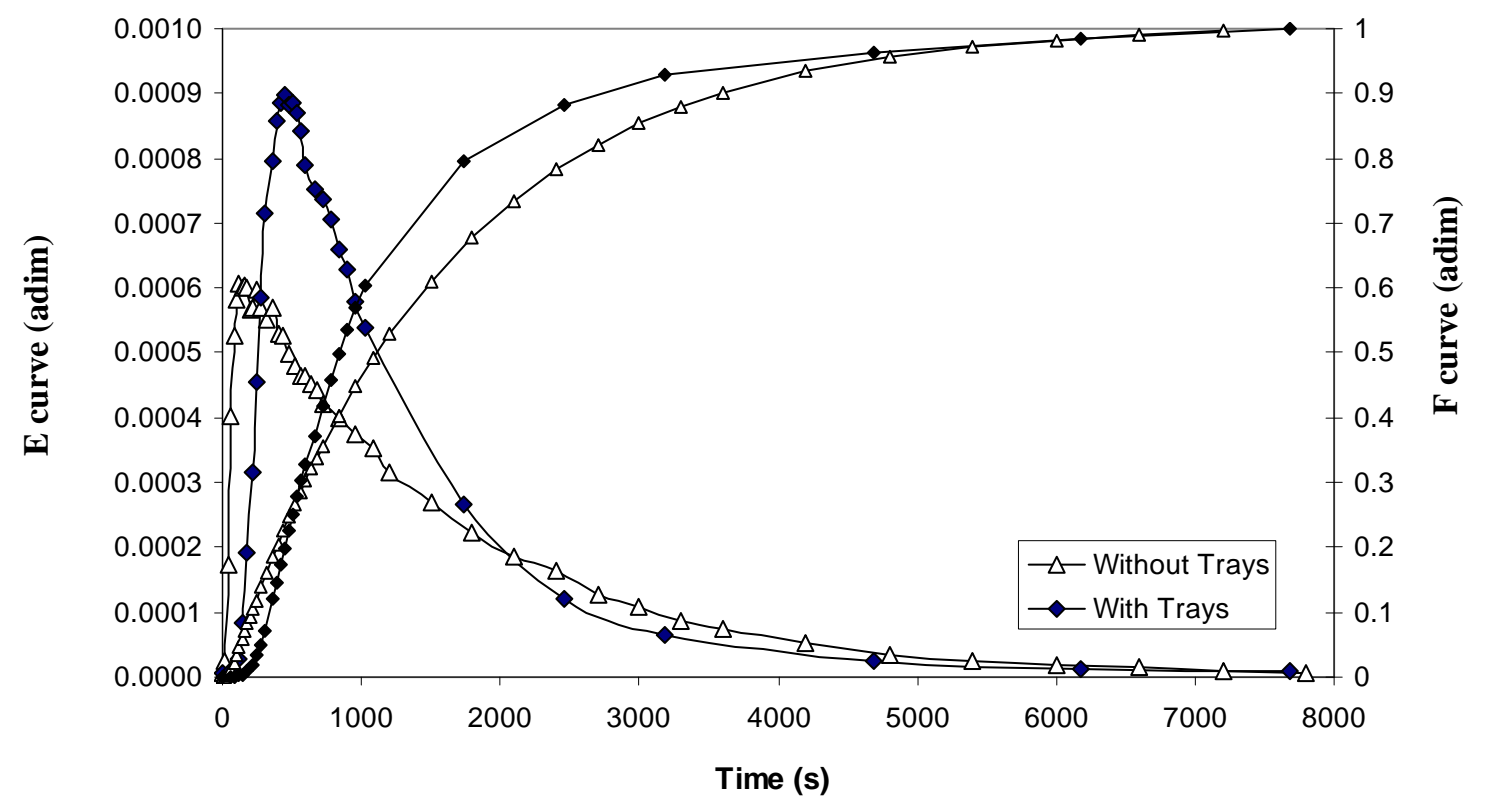

Fig. 13 E and F curves for air-light oil systems at $35 \mathrm{l} / \mathrm{h}$ of liquid flow rate with and without trays. 


\section{Conclusions}

Without internal trays inside the column was found that gas hold up increase along the reactor. It was possible to identify heterogeneous bubble, transition and turbulent flow pattern areas at different superficial gas velocities for the air-light oil system.

It was also determine that average gas bubble size increase along the reactor at bubble regime from 2-5 $\mathrm{mm}$ but at turbulent regime, stay oscillating between 1-3 mm.

At the same reactor height level, average gas bubble size decreases with the superficial gas velocity.

Average interfacial area increases exponentially with gas superficial velocity at any reactor height, till $1,412 \mathrm{~m}^{2} / \mathrm{m}^{3}$ for the air-light oil system but, at bubble flow regime, the average interfacial area is lower than $100 \mathrm{~m}^{2} / \mathrm{m}^{3}$, that perhaps negatively impact the reactor performance.

Internal plates in the reactor always increase global gas hold up at any condition or system used. Residence time distributions curves, Péclet numbers and dispersion coefficients founded, show that this reactor with internal plates used here still tends to be a complete mixing reactor under the operating conditions used. The new RTD curve was displaced on time because the death space created by the internal inside the column

\section{Acknowledgments}

The authors thank William Jimenez and David Mendoza from Intevep for providing technical support on the video and photographic section used in this work. We thank Wilfredo Mendoza from Intevep for the aid provided on the tracer definition and chemical measurements.

\section{References}

[1] Sardella, R., Rojas, J. D., Zacarias, L., Delgado, O., Rivas A., López E., and Marténez S. 2009. “Transportable Upgraded Crude: Aquaconversion ${ }^{\circledR}$ Technology Overview." World Heavy Oil Congress, Margarita Island,
Venezuela.

[2] Akita, K., and Yoshida, F. 1973. "Gas Hold-up and Volumetric Mass Transfer Coefficient in Bubble Columns." Ind. Eng. Chem. Process Des. Develop 12: 76-8.

[3] Hoefsloot, H., and Krishna, R. 1993. "Influence of Gas Density on the Stability of Homogeneous Flow in Bubble Columns." Ind. Eng. Chem. Res. 32 (4): 747-50.

[4] Lenvenspiel, O. 1981. Chemical Reaction Engineering, Second Edition, Reverté s.a. Editorial.

[5] Duns, H. J. R., and Ros, N. C. J. 1983. "Vertical Flow of Gas Liquid Mixtures from Boreholes." Proceedings of 6th World Petroleum Congress, 451-4.

[6] Carberry, J. "Chemical and Catalytic Reaction Engineering." First Edition, McGraw-Hill Chemical Engineering Series, New York, 149-51.

[7] Daly, J., Patel, S., and Bukur, D. 1992. "Measurement of a Gas Hold Ups and Sauter Mean Bubble Diameter in Bubble Column Reactor by Dynamic Disengagement Method." Chemical Engineering Science 47 (13/14): 3647-54.

[8] Shah, Y. T., Kelkar, B. G., and Godbole, S. P., 1982. "Design Parameters Estimations for Bubble Column Reactors." AlChe Journal 28: 353-60.

[9] Sardella, R., Zacarías, L., Paiva, M., and Medina, H. 2011. "Bubble Column Reactor Fluid Dynamic Study at Pilot Plant Scale for Residue and Extra-heavy Crude Oil Upgrading Technology." World Heavy Oil Congress, Edmonton, Alberta Canada.

[10] Billet, A. M., Chaumat, H, and Delmas, H. "Hydrodynamics and Mass Transfer in Bubble Column: Influence of Liquid Phase Surface Tension." Chemical Engineering Science 62: 7378-90.

[11] Pino, L. Z., Yepez, M. M., and Saes, A. E. 1990. "An Experimental Study of Gas Holds Up in Two-phase Bubble Columns with Foaming Liquids." Chem. Eng. Comm 89: 155-75.

[12] Guitian, J., and Joseph, D. 1998. "How Bubbly Mixtures Foam and Foam Control Using Fluidized Bed." Ind. J. Multiphase Flow 2 (1): 1-16.

[13] Morsi, B., Behkish, A., Men, Z. and Inga, J. 2002. "Mass Transfer Characteristics in a Large-scale Slurry Bubble Columns Reactor with Organic Liquid Mixtures." Chemical Engineering Science 57 (16): 3307-24.

[14] Soong, Y., Harke, F. W., Gamwo I. K., Schehl, R. R. and. Zarochak, M. F. 1997. "Hydrodynamic Study in a Slurry Bubble Column Reactor." Catalyst Today 35: 427-34.

[15] Lemoine, R., Behkish, A., and Morsi, B. 2004. "Hydrodynamic and Mass-transfer Characteristics in 


\section{Extra-heavy Crude Oil Upgrading Technology}

Organic Liquid Mixtures in a Large-scale Bubble Column Reactor for the Toluene Oxidation Process." Ind. Eng. Chem. Res. 43: 6195-212.

[16] Trambouze, P., and Euzen, J. P. 2002. Chemical Reactor from Design to Operation, Edición Technip, 204-15.

[17] Kelkar, B., Shah, Y., and Carr, N. 1984. "Hydrodynamics and Axial Mixing in a Three Phase Bubble Column.
Effects of Slurry Properties." Ind. Eng. Chem. Process Des. Dev. 23 (2): 308-13.

[18] Alvaré, J. and Al-Dahhan, M. 2006. "Gas Holdup in Trayed Bubble Column Reactor." Ind. Eng. Chem. Res. 45: 3320-6.

[19] Fogler, S. 2001. Elements of Chemical Reaction Engineering Prentice Hall. Mexico: D.F. 